\title{
HADRONIC CHARM DECAYS IN CLEO
}

\author{
STEVEN R. BLUSK \\ Department of Physics, Syracuse University, Syracuse, NY 13244 \\ E-mail:sblusk@phy.syr.edu
}

\begin{abstract}
We present several results on hadronic $D$ meson decays. We report on results from the scan of the energy region from $3970 \mathrm{MeV}$ to $4260 \mathrm{MeV}$, which was used to determine an optimal energy to carry out the $D_{s}$ physics program of CLEO-c. Improved measurements of inclusive and exclusive $D$ and $D_{s}$ branching fractions are presented. We also show results on Dalitz analyses of $D^{+} \rightarrow \pi^{+} \pi^{-} \pi^{+}$ using CLEO-c data and $D^{0} \rightarrow K^{+} K^{-} \pi^{0}$ using CLEO III data.
\end{abstract}

Keywords: Charm; Hadronic; Dalitz.

\section{Introduction}

The study of leptonic and semileptonic charm decays provide direct access to CKM elements, decay constants and form factors. Measurements of hadronic branching fractions (BF) and their Dalitz structure provide important inputs to $B$-physics measurements undertaken at B-factories. CLEO has collected $281 \mathrm{pb}^{-1}$ of data at the $\psi(3770)$ and, as of this time, an additional $\sim 350 \mathrm{pb}^{-1}$ at $\sqrt{s}=4170 \mathrm{MeV}$. In this report, we describe several recent hadronic charm analyses from CLEO-c, and one from CLEO-III $(\sqrt{s} \approx 10 \mathrm{GeV})$.

\section{The $D_{s}$ Energy Scan}

CLEO scanned the energy region from 3970$4260 \mathrm{MeV}$ with the primary goal of determining an optimal energy for $D_{s}$ physics. The scan included 12 points with a total luminosity of about $60 \mathrm{pb}^{-1}$. Candidate $D^{0}$, $D^{+}$and $D_{s}$ mesons are reconstructed in 3, 5 , and 8 modes, respectively. Because of the highly constrained kinematics, the momentum of the candidate can be used to determine the final state, e.g., $D \bar{D}, D^{*} \bar{D}, D^{*} \bar{D}^{*}$, $D_{s} \bar{D}_{s}$, or $D_{s}^{*} \bar{D}_{s}$.

We measure charm cross-sections in three ways: (1) inclusive hadronic event counting, with the non-charm components subtracted, (2) inclusive $D_{(s)}$ counting, and
(3) sum of $D_{(s)}^{(*)} \bar{D}_{(s)}^{(*)}$ exclusive final states. We show in Fig. 1 the $D \bar{D}$ (top) and $D_{s} \bar{D}_{s}$ (bottom) cross-sections using exclusive final states. A peak cross-section of about $0.9 \mathrm{nb}$ for $D_{s}^{*} \bar{D}_{s}$ is observed near $4170 \mathrm{MeV}$, which was then selected as the optimal energy for the CLEO-c $D_{s}$ physics program.

The total charm cross-sections obtained using the three different methods are compared in Fig. 2 (top). The inclusive measurements show a clear excess over exclusive reconstruction of $D_{(s)}^{(*)} \bar{D}_{(s)}^{(*)}$. Much of this excess can be attributed to a $D^{*} \bar{D} \pi$ final state. We model this excess as a non-resonant contribution, and show in Fig. 2 (bottom) the momentum spectrum of $D^{0} \rightarrow K^{-} \pi^{+}$decays using $178 \mathrm{pb}^{-1}$ of data at $E_{\mathrm{cm}}=4170 \mathrm{MeV}$, compared to a simulation which includes the multi-body contribution. The prominent peaks from two-body decays are indicated along with the broader spectrum from $\bar{D}^{*} D \pi$. With the three-body final state included, a good description of the data is obtained.

\section{Hadronic Branching Fractions}

Production of $D \bar{D}$ and $D_{s} \bar{D}_{s}$ allows CLEO-c to determine absolute BF's as well as $N_{D \bar{D}}$ using yields of double-tagged and singletagged events. Results based on $57 \mathrm{pb}^{-1}$ 

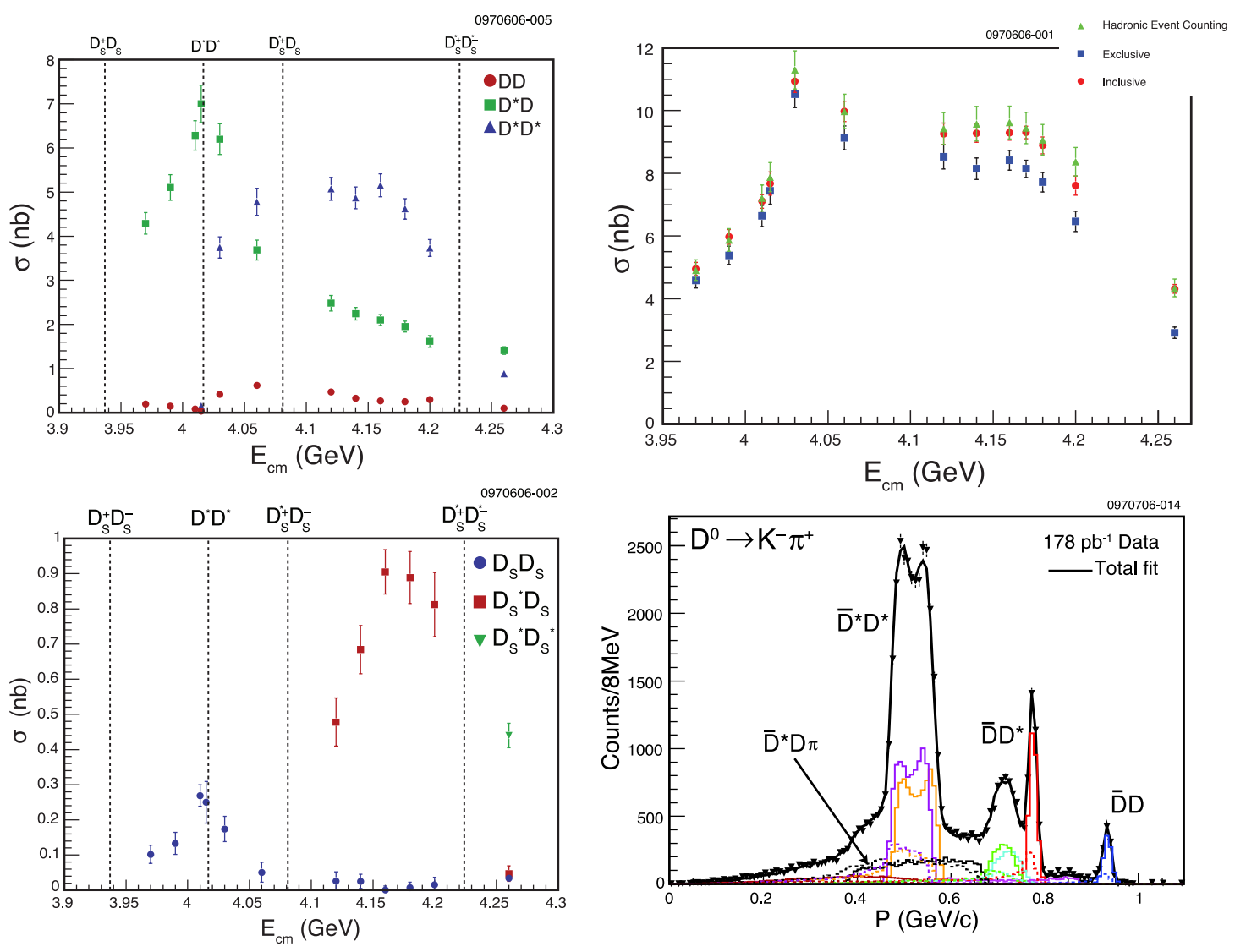

Fig. 1. Measured production cross-section for $D \bar{D}$, $D \bar{D}^{*}$, and $D^{*} \bar{D}^{*}$ (top) and $D_{s} \bar{D}_{s}, D_{s} \overline{D_{s}^{*}}$, and $D_{s}^{*} \overline{D_{s}^{*}}$ (bottom) l) as a function of $\sqrt{s}$. The various $D_{(s)}$ pair thresholds are indicated.

have already been published $^{2}$, yielding $D$ BF's in Cabibbo-favored modes which are competitive with or better than the world average. Results based on $281 \mathrm{pb}^{-1}$ are imminent, and should provide uncertainties at the level of $1.5 \%$ on the key normalization modes: $D^{0} \rightarrow K^{-} \pi^{+}$and $D^{+} \rightarrow K^{-} \pi^{+} \pi^{+}$.

Measurements of Cabibbo-suppressed decays using $281 \mathrm{pb}^{-1}$ have also been published $^{3}$. Six new decay modes and eight improved measurements were reported. Improved $D \rightarrow \pi \pi \mathrm{BF}$ measurements were used to perform an isospin analysis, yielding an amplitude ratio $A_{2} / A_{0}=0.420 \pm 0.014 \pm$ 0.016 and strong phase shift $\delta_{I}=(86.4 \pm 2.8 \pm$ $3.3)^{\circ}$ between the $\Delta I=3 / 2$ and $\Delta I=1 / 2$

Fig. 2. (Top) Measured charm cross-section using hadron counting, inclusive $D_{(s)}$ yields and exclusive $D_{(s)}^{(*)} \bar{D}_{(s)}^{(*)}$ final states. (Bottom) Momentum spectrum of $D^{0}$ mesons at $E_{\mathrm{cm}}=4160 \mathrm{MeV}$ in data (points) and simulation (lines).

isospin amplitudes.

We also report on preliminary measurements of BF's in $D_{s}$ decays using $\sim 200 \mathrm{pb}^{-1}$ of data collected at $E_{\mathrm{cm}}=4170 \mathrm{MeV}$. As with the $D$ hadronic analysis ${ }^{2}$, we use yields from single-tag modes $\left(D_{s} \rightarrow\right.$ $K_{S} K^{+}, K^{+} K^{-} \pi^{+}, K^{+} K^{-} \pi^{+} \pi^{0}, \pi^{+} \pi^{-} \pi^{+}$, $\left.\eta \pi^{+}, \eta^{\prime} \pi^{+}\right)$and 36 double-tag modes to fit for the absolute $D_{s}$ BF's. Table 1 shows the single-tag yields, average $D_{s}$ efficiencies and the BF's from the fit (double-tag yields not shown). While these results are preliminary, the uncertainties are already significantly smaller than the 2006 world averages. The decay $D_{s} \rightarrow \phi \pi$ is a sub-mode 
of $K^{+} K^{-} \pi^{+}$, and exraction of its branching fraction is complicated due to interference with the nearby $f_{0}$. Alternately, we measure the partial BF, consisting of events with $M_{K^{+} K^{-}}-M_{\phi}<10 \mathrm{MeV}$. The resulting partial $\mathrm{BF} \mathcal{B}_{M_{K^{+} K^{-}}-M_{\phi}<10 \mathrm{MeV}}\left(D_{s} \rightarrow\right.$ $\left.K^{+} K^{-} \pi\right)$ is $(1.98 \pm 0.12 \pm 0.09) \%$.

\section{Dalitz Analyses}

Multi-body decays are often dominated by, or have significant contributions from one or more quasi-twobody decays. Dalitz analyses allow for extraction of these contributing amplitudes and relative strong phases by constructing a total amplitude which is a coherent sum of complex amplitudes ${ }^{5}$.

We first report on a CLEO Dalitz analTable 1. Preliminary results of $D_{s}$ branching frac- ysis of the decay $D^{+} \rightarrow \pi^{+} \pi^{-} \pi^{+6}$. This tions based on $\sim 200 \mathrm{pb}^{-1}$ of data collected at work is fueled by a number of experimental $E_{\mathrm{cm}}=4170 \mathrm{MeV}$. Yields are the sum of $D_{s}^{+}$and $D_{s}^{-}$.

\begin{tabular}{lccc}
\hline $\begin{array}{l}\text { Mode } \\
D_{s}^{ \pm} \rightarrow\end{array}$ & Yield & $\begin{array}{c}\text { Eff. } \\
(\%)\end{array}$ & $\begin{array}{c}\text { BF } \\
(\%)\end{array}$ \\
\hline$K_{S} K^{ \pm}$ & $1983 \pm 54$ & 37.5 & $1.50 \pm 0.09 \pm 0.05$ \\
$K^{+} K^{-} \pi^{ \pm}$ & $8666 \pm 126$ & 44.3 & $5.57 \pm 0.30 \pm 0.19$ \\
$K^{+} K^{-} \pi^{ \pm} \pi^{0}$ & $2410 \pm 119$ & 12.5 & $5.62 \pm 0.33 \pm 0.51$ \\
$\pi^{ \pm} \pi^{\mp} \pi^{ \pm}$ & $1916 \pm 112$ & 49.4 & $1.12 \pm 0.08 \pm 0.05$ \\
$\eta \pi^{ \pm}$ & $1117 \pm 70$ & 19.5 & $1.47 \pm 0.12 \pm 0.14$ \\
$\eta^{\prime} \pi^{ \pm}$ & $733 \pm 33$ & 5.4 & $4.02 \pm 0.27 \pm 0.30$ \\
\hline
\end{tabular}
results $^{7,8}$ that support the existence of a low mass scalar state, referred to as the $\sigma$. FOCUS, which employs a $K$ matrix formalism ${ }^{9}$, does not require the inclusion of the $\sigma$ to describe the low $\pi \pi$ mass region in the $D \rightarrow$ $\pi^{+} \pi^{-} \pi^{+}$decay.

CLEO-c's $D^{+} \rightarrow \pi^{+} \pi^{-} \pi^{+}$sample is comprised of about $2600 \mathrm{D}^{+} \rightarrow \pi^{+} \pi^{-} \pi^{+}$decays and about 2200 background events after vetoing events consistent with $K_{S}^{0} \rightarrow \pi^{+} \pi^{-}$. Several $\pi^{+} \pi^{-}$resonant contributions are included in the fit, including $\rho(770), \rho(1450)$, $f_{0}(980), f_{2}(1270), f_{0}(1370), f_{0}(1500)$, and $\sigma$. The $\sigma$ is modeled as a pole in the complex plane ${ }^{10}$ and the $f_{0}(980)$ using the Flatté parameterization. The resonance parameters are taken from previous experiments ${ }^{11,12}$. The resulting fit fractions and relative phases are shown in Table 3. Our data are in general agreement with those of E791 ${ }^{7}$. In both cases, neglecting this low mass contribution gives a poor description of the data. Alternate descriptions of the low mass $\pi \pi$ region are being investigated.

CLEO has also performed a Dalitz analysis of the $D^{0} \rightarrow K^{+} K^{-} \pi^{0}$ decay $^{13}$ using $\sim 9 \mathrm{fb}^{-1}$ of data on/just below the $\Upsilon(4 \mathrm{~S})$. This final can be used to extract the CPviolating angle, $\gamma$, through the interference of $B^{+} \rightarrow D^{0} K^{+}$and $B^{+} \rightarrow \bar{D}^{0} K^{+14}$. We reconstruct and tag the flavor of $D^{0}$ mesons via the sequential decay $D^{*+} \rightarrow D^{0} \pi^{+}$. A sample of 735 events are selected, of which about 130 are background. The signal is mod- $s \bar{s}$ component of $\eta, \eta^{\prime}$, and $\phi$ mesons lead to a larger production rate in $D_{s}$ decays. 
Table 2. Inclusive branching fractions for $D^{0}, D^{+}$and $D_{s}$ to $\eta, \eta^{\prime}$, and $\phi$ mesons (in percent).

\begin{tabular}{cccc}
\hline & $\eta X$ & $\eta^{\prime} X$ & $\phi X$ \\
\hline$D^{0}$ & $9.5 \pm 0.4 \pm 0.8$ & $2.48 \pm 0.17 \pm 0.21$ & $1.05 \pm 0.08 \pm 0.07$ \\
$D^{+}$ & $6.3 \pm 0.5 \pm 0.5$ & $1.04 \pm 0.16 \pm 0.09$ & $1.03 \pm 0.10 \pm 0.07$ \\
$D_{s}$ & $23.5 \pm 3.1 \pm 2.0$ & $8.7 \pm 1.9 \pm 0.8$ & $16.1 \pm 1.2 \pm 1.1$ \\
\hline
\end{tabular}

Table 3. Preliminary results on the Dalitz analysis of the $D^{+} \rightarrow \pi^{+} \pi^{-} \pi^{+}$decay showing the fit fraction $(\mathrm{FF})$ and phase relative to $\rho(770) \pi^{+}$. CLEO limited are at the $90 \%$ confidence level. We also show the corresponding fit fractions from E791.

\begin{tabular}{lccc}
\hline Mode & CLEO FF & Phase $\left(^{\circ}\right)$ & E791 FF \\
\hline$\rho(770) \pi^{+}$ & $20.0 \pm 2.5$ & 0(fixed) & $33.6 \pm 3.9$ \\
$\sigma \pi^{+}$ & $41.8 \pm 2.9$ & $-3 \pm 4$ & $46.3 \pm 9.1$ \\
$f_{2}(1270) \pi^{+}$ & $18.2 \pm 2.7$ & $-123 \pm 6$ & $19.4 \pm 2.5$ \\
$f_{0}(980) \pi^{+}$ & $4.1 \pm 0.9$ & $12 \pm 11$ & $6.2 \pm 1.4$ \\
$f_{0}(1500) \pi^{+}$ & $3.4 \pm 1.3$ & $-44 \pm 21$ & - \\
Non-resonant & $<3.5$ & - & $7.8 \pm 6.6$ \\
$\rho(1450) \pi^{+}$ & $<2.4$ & - & $0.7 \pm 0.8$ \\
\hline
\end{tabular}

eled with three Breit-Wigner contributions, $D^{0} \rightarrow \phi \pi^{0}, K^{*+} K^{-}$, and $K^{*-} K^{+}$, along with a non-resonant (NR) interfering component. The background shape is determined by a fit to the Dalitz plot obtained from $D^{0}$ candidates in the $\Delta M=M\left(D^{*+}\right)-M\left(D^{0}\right)$ sideband region. The amplitudes, phases and fit fractions are shown in Table 4. For $K^{*-} K^{+}$, which is most relevant for this analysis, the uncertainties include both statistical and systematic sources; the others are only statistical. The amplitude ratio and relative phase between $K^{*-} K^{+}$and $K^{*+} K^{-}$ are thus found to be: $\left|A_{K^{*-} K^{+}} / A_{K^{*+} K^{-}}\right|=$ $0.52 \pm 0.05 \pm 0.04$ and $\delta_{K^{*-} K^{+}-K^{*+} K^{-}}=$ $(332 \pm 8 \pm 11)^{\circ}$. This is the first measurement of this phase shift, and the amplitude ratio measurement is a significant improvement over previous estimates using $D^{0} \rightarrow K^{*} K$ branching fractions.

We gratefully acknowledge the effort of the CESR staff in providing us with excellent luminosity and running conditions, and the National Science Foundation for support of this work.
Table 4. Dalitz results of the $D^{+} \rightarrow K^{+} K^{-} \pi^{0}$ decay showing the amplitudes, phases and fit fractions (FF) relative to $K^{*+} K^{-}$.

\begin{tabular}{lccc}
\hline Mode & Amplitude & Phase $\left(^{\circ}\right)$ & FF \\
\hline$K^{*+} K^{-}$ & 1.0 & 0(fixed) & $46.1 \pm 3.1$ \\
$K^{*-} K^{+}$ & $0.52 \pm 0.06$ & $332 \pm 14$ & $12.3 \pm 2.2$ \\
$\phi \pi^{0}$ & $0.64 \pm 0.04$ & $326 \pm 9$ & $1.94 \pm 1.6$ \\
$\mathrm{NR}$ & $5.62 \pm 0.45$ & $220 \pm 5$ & $36.0 \pm 3.7$ \\
\hline
\end{tabular}

\section{References}

1. B. Aubert et. al. (BaBar Collaboration), Phys. Rev. Lett. 95, 142001 (2005).

2. Q. He et al. (CLEO Collaboration), Phys. Rev. Lett. 95, 121801 (2005).

3. P. Rubin et al. (CLEO Collaboration), Phys. Rev. Lett. 96, 081802 (2006).

4. G. S. Huang et al. (CLEO Collaboration), submitted to Phys. Rev. D (CLNS 06-1975).

5. W. M. Yao et. al., J. Phys. G 33, 1 (2006).

6. M. Dubrovin, Proceedings of the CHARM 2006, June 6, 2006, Beijing, China [hepex/0608008]; D. Cinabro, Flavor Physics and CP Violation, April 12, 2006, Vancouver, Canada [hep-ex/0605074].

7. E. M. Aitala et al. (E791 Collaboration), Phys. Rev. Lett. 86, 770 (2001).

8. M. Ablikim et al. (BES Collaboration), Phys. Lett. B 598, 149 (2004).

9. P. L. Frabetti et al. (FOCUS Collaboration), Phys. Lett. B 585, 200 (2004).

10. J. A. Oller and E. Oset, Nucl. Phys. A 620 438, (1997) [hep-ph/9702314].

11. S. Eidelman, et. al., Phys. Lett. B 592, 1 (2004)

12. M. Ablikim et al. (BES Collaboration), Phys. Lett. B 607, 243 (2005).

13. C. Cawlfield et al. (CLEO Collaboration), Phys. Rev. D 74, 031108 (2006).

14. Y. Grossma,, Z. Ligeti, and A. Soffer, Phys. Rev. D 67, 071301 (2003). 